Revue d'histoire de l'Amérique française

REVUE D.HISTOIRE DE L'AMÉRIQUE FRANÇAISE

\title{
Les rituels du mariage dans la vallée du Haut-Richelieu : comparaison ville/campagne $\mathrm{au} \mathrm{XX}^{\mathrm{e}}$ siècle1
}

\section{Martine Tremblay}

Volume 54, numéro 3, hiver 2001

URI : https://id.erudit.org/iderudit/005623ar

DOI : https://doi.org/10.7202/005623ar

Aller au sommaire du numéro

Éditeur(s)

Institut d'histoire de l'Amérique française

ISSN

0035-2357 (imprimé)

1492-1383 (numérique)

Découvrir la revue

Citer cet article

Tremblay, M. (2001). Les rituels du mariage dans la vallée du Haut-Richelieu : comparaison ville/campagne au $\mathrm{Xx}^{\mathrm{e}}$ siècle1. Revue d'histoire de l'Amérique française, 54(3), 385-410. https://doi.org/10.7202/005623ar
Résumé de l'article

Les rituels du mariage sont utilisés comme des indicateurs permettant de saisir quelques modalités du changement culturel au $\mathrm{XX}^{\mathrm{e}}$ siècle. Grâce à une enquête orale menée auprès de témoins mariés au cours des périodes 1920-1940 et 1980-1995 dans la région de Saint-Jean-Iberville (Québec), il est possible d'observer la diffusion des innovations à travers l'espace social. Si l'expansion du mode de vie urbain a entraîné, de manière globale, la transformation des pratiques rituelles, certaines coutumes de la campagne ont été conservées par les familles de la ville. Plus intéressant encore, cette enquête a permis de démontrer que des pratiques paysannes anciennes, mises à l'écart pendant un certain temps, sont redécouvertes et réactualisées par les couples mariés à la fin du $\mathrm{XX}^{\mathrm{e}}$ siècle. La recherche sur les échanges culturels entre la ville et la campagne ouvre sur des perspectives inattendues.
Tous droits réservés @ Institut d'histoire de l'Amérique française, 2001
Ce document est protégé par la loi sur le droit d'auteur. L'utilisation des services d'Érudit (y compris la reproduction) est assujettie à sa politique d'utilisation que vous pouvez consulter en ligne.

https://apropos.erudit.org/fr/usagers/politique-dutilisation/ 


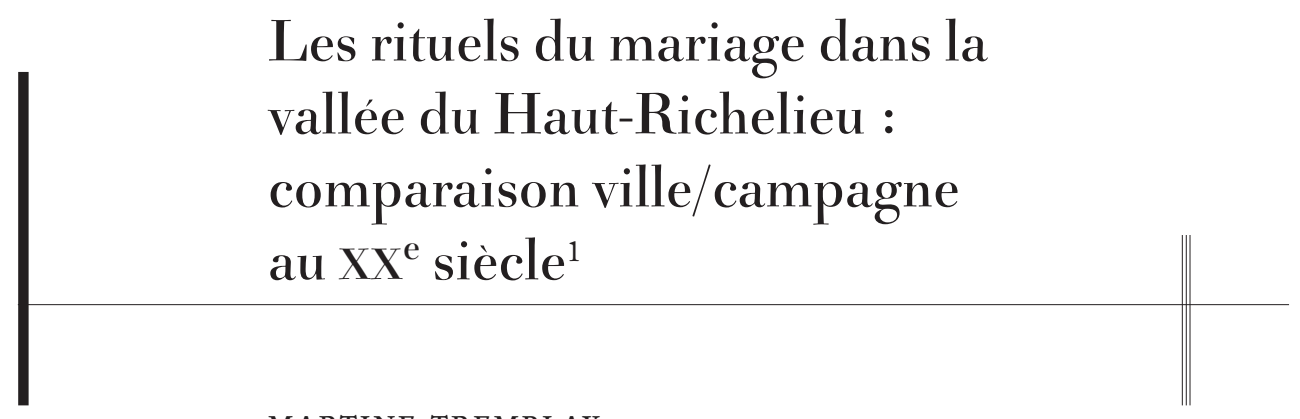

MARTINE TREMBLAY

INRS - Culture et Société

RÉSUMÉ - Les rituels du mariage sont utilisés comme des indicateurs permettant de saisir quelques modalités du changement culturel au $X X^{\mathrm{e}}$ siècle. Grâce à une enquête orale menée auprès de témoins mariés au cours des périodes 1920-1940 et 1980-1995 dans la région de Saint-Jean-lberville (Québec), il est possible d'observer la diffusion des innovations à travers l'espace social. Si l'expansion du mode de vie urbain a entraîné, de manière globale, la transformation des pratiques rituelles, certaines coutumes de la campagne ont été conservées par les familles de la ville. Plus intéressant encore, cette enquête a permis de démontrer que des pratiques paysannes anciennes, mises à l'écart pendant un certain temps, sont redécouvertes et réactualisées par les couples mariés à la fin du XX $X^{\mathrm{e}}$ siècle. La recherche sur les échanges culturels entre la ville et la campagne ouvre sur des perspectives inattendues.

ABSTRACT - Marriage rituals are used here as indicators of the cultural change which occurred in the $\mathrm{XX}^{\text {th }}$ century. Through an oral survey involving people married during the periods 1920-1930 and 1980-1995 in Saint-Jean-lberville (Québec) it is possible to observe the spreading of innovation in the social space. If the expansion of the urban mode of living transformed ritual practices in general, it so happens that some customs of the countryside were maintained by families in the city. More interestingly, this inquiry allowed us to demonstrate that some ancient rural practices, put aside for some time, were rediscovered and renewed by couples married at the end of the $X X^{\text {th }}$ century. Research into cultural exchanges between the city and the country opens up unexpected perspectives.

1. Les résultats de la recherche présentés ici sont tirés de ma thèse de doctorat. Je tiens à remercier chaleureusement mes directeur et codirecteur de thèse, René Hardy et Gérard Bouchard. 
L

E CHAMP DE L'HISTOIRE de la culture a bénéficié, ces dernières années, en portant le regard sur la variabilité des formes rituelles dans le temps et dans l'espace, ainsi qu'en fonction de la position sociale des acteurs. Notre enquête menée dans la vallée du Haut-Richelieu a permis d'apprécier la diffusion des innovations culturelles en deux moments précis au $\mathrm{xx}^{\mathrm{e}}$ siècle. Les rituels du mariage ont été observés entre 1920 et 1940, dans un contexte d'intense urbanisation et de différenciation sociale, afin d'évaluer les distinctions ville/campagne et socioprofessionnelles. Une autre prise de vue a été réalisée entre 1980 et 1995, afin de constater s'il subsiste des écarts dans les pratiques rituelles à la fin $\mathrm{du} \mathrm{xx}^{\mathrm{e}}$ siècle. Dans le cadre restreint de cet article, nous présentons uniquement les résultats de la recherche qui concernent les rapports ville/ campagne. Auparavant, nous allons esquisser les bases conceptuelles et méthodologiques sur lesquelles repose cette recherche.

\section{LES RITUELS COMME INDICATEURS DE LA DYNAMIQUE CULTURELLE}

Nous considérons les rituels du mariage comme des indicateurs devant permettre de comprendre certains processus du changement culturel ${ }^{3}$. Les rituels sont analysés dans leur morphologie, c'est-à-dire dans leur matérialité visible et perceptible, dans ce qu'il est possible d'observer, plutôt que dans leur signification. La question du sens des rites n'est toutefois pas écartée. L'analyse conduit à situer ces gestes dans leur contexte social d'exécution : les rites signifient la transformation du lien familial et la constitution du lien conjugal.

Les rituels sont observés ici comme des manifestations de la culture, plus précisément de la culture instituée qui est celle de la reproduction et des usages, par opposition à la culture instituante qui est celle de l'invention et de la production ${ }^{4}$. La ritualité est un mode de connaissance et d'action

2. Le groupe de recherche sur les dynamiques culturelles interrégionales de l'IREP a réalisé une enquête sur les rituels du mariage dans plusieurs régions du Québec. Nos travaux, bien que distincts, s'inscrivent dans le cadre de cette recherche.

3. Gérard Bouchard a été le premier à faire usage des rituels pour observer les dynamiques culturelles. Voir Gérard Bouchard, Josée Gauthier et Marie-Josée Huot, «Permanences et mutations dans l'histoire de la culture paysanne québécoise", dans G. Bouchard avec la collaboration de S. Courville, La construction d'une culture. Le Québec et l'Amérique française (Sainte-Foy, Les Presses de l'Université Laval, 1993), 261-305.

4. Gérard Bouchard, «L'analyse morphologique de la culture : l'exemple des rituels matrimoniaux», Ethnologie française, 29,4 (1999) : 639-652. 
basé essentiellement sur la reproduction de gestes dont l'usage, plus ou moins ancien, prouve l'efficacité. Les rituels expriment les fondements de la culture par des mots et des gestes en même temps qu'ils renouvellent les valeurs et les croyances en les actualisant. Le couple culture instituée/culture instituante ne doit pas être considéré comme figé. Le rapport au monde, caractéristique de la culture instituée, n’est pas fondé de manière univoque sur la tradition, bien qu'il repose davantage sur des éléments connus et réarrangés. La ritualité est d’abord faite de répétitions, mais l'adaptation des actions individuelles au jeu des forces sociales peut entraîner une transformation fondamentale des rituels.

L'analyse des rituels du mariage a jusqu'à maintenant reposé sur le concept de rites de passage tel que défini au début du siècle par le folkloriste français Arnold Van Gennep, dont les écrits ont marqué l'étude de la ritualité. Van Gennep reprenait la division durkheimienne du monde en deux sphères : le sacré et le profane ${ }^{5}$. Il affirmait que l'union du couple constituait une transgression de l'ordre sacré parce qu'elle impliquait de multiples changements dans la famille, les groupes d'appartenance et la communauté villageoise ${ }^{6}$. Ce passage du monde des jeunes célibataires au groupe des adultes mariés recouvrait une transition, plus ou moins ritualisée, qui rendait l'union sexuelle et la procréation acceptables pour la société. À la fin du $\mathrm{xx}^{\mathrm{e}}$ siècle, le mariage ne correspond plus à un changement de statut, il n'est plus essentiel à la vie maritale ni à la fondation d'une famille. On ne peut plus le considérer comme un rite de passage de la manière dont Van Gennep définissait ce dernier. Par contre, les jeunes qui, aujourd'hui, décident de formaliser leur union par un rituel comportant une cérémonie religieuse adhèrent à une vision sacrée du mariage.

La définition du rituel adoptée ici tente de rendre compte de cet effacement (ou étalement) du "passage » et d'inclure à la fois les rites religieux et les rites qui relèvent de la sociabilité familiale et entre jeunes. Le rituel est un ensemble de gestes (rites, pratiques, coutumes) faits par les individus dans certaines circonstances ou en rapport avec certains événements, correspondant, avec un écart plus ou moins grand selon les individus et les époques, à la norme ou à la mode. Cette action sociale est construite sur la ligne de tension qui relie l'individu à la société : elle révèle du même coup comment l'individu s'intègre à la société et comment se

5. Arnold Van Gennep, Les rites de passage. Étude systématique des rites (Paris, A. et J. Picard, 1981), 16.

6. Ibid., 176-177. 
réalise concrètement sa socialisation. La mise en scène des rituels par les acteurs dans un temps et un espace précis reconduit l'univers de sens et réactualise les valeurs largement partagées.

L'acte social n'est pas un geste purement intentionnel comme il n'est pas non plus entièrement déterminé socialement ${ }^{7}$. Certains groupes, à une époque donnée, peuvent bénéficier d'une plus grande latitude. Leur rituel se rapproche alors de la mode ou de la norme qui entraîne mais ne contraint pas, reposant davantage sur la conviction que sur l'obligation, bien que la mode et la norme puissent émaner d'institutions ou de groupes formels. À d'autres moments de l'histoire, le rituel est prescrit et oblige l'adhésion. Il est alors très près de la norme imposée par le groupe d'appartenance (la famille, le groupe des jeunes) ou par certaines institutions (comme l'État et l'Église), et cette norme devient pratiquement incontournable. Le rituel est donc un geste individuel dont la signification est construite socialement. Interface entre l'individu et la société, le rituel réactualise la cohésion sociale de manière incessante ${ }^{8}$.

\section{LE MARIAGE À LA CAMPAGNE ET À LA VILLE}

L'une des voies de pénétration de la culture que nous avons empruntée tient compte du milieu de vie des individus, soit la ville ou la campagne. Notre hypothèse est que les individus ont réaménagé les rituels du mariage à mesure que se sont transformées leurs conditions de vie : le mode de vie urbain a favorisé de plus fréquentes occasions de rencontre entre personnes non apparentées et des rapports différents entre hommes et femmes. La ville est aussi le lieu où s'est intensifiée la différenciation sociale issue de la division du travail, ouvrant un espace possible à l'expression de pratiques distinctives.

Arnold Van Gennep a dénié l'authenticité des rituels en milieu urbain?. La ville et le travail impliquant des machines éloigneraient des croyances magiques et de la vision sacrée liée à la pratique de l'agriculture. Les rituels observés à la ville seraient donc des survivances rattachées à certains groupes d'artisans et viseraient d'autres objectifs que celui d'appréhender les grandes manifestations naturelles de l'univers. En associant la création rituelle à la vie paysanne, Van Gennep disqualifiait pour longtemps l'étude

7. Pierre Bourdieu, Le sens pratique (Paris, Éditions de Minuit, 1980), 112; Erving Goffman, La mise en scène de la vie quotidienne, 1 : La présentation de soi (Paris, Éditions de Minuit, 1973), 18.

8. Sur le rôle du rituel dans le maintien de l'ordre social : Pierre Bourdieu, op. cit., 97.

9. Arnold Van Gennep, Manuel de folklore français contemporain, I : Introduction générale et première partie : du berceau à la tombe (Paris, A. et J. Picard, 1972), 56. 
des rituels urbains. Si un peu plus loin dans le même ouvrage il a nuancé sa position, les chercheurs français ont continué d'associer dynamisme de la ritualité et paysannerie. La recherche au Québec a aussi été marquée par cette conception des rituels. Après les travaux des folkloristes ÉdouardZotique Massicotte et sœur Marie-Ursule, qui ont consigné ce qu'ils considéraient comme les dernières manifestations des rituels paysans, l'étude des rituels du mariage a été délaissée ${ }^{10}$. Ni les ethnologues, ni les anthropologues, ni les historiens n'ont repris la question avant les années 1980.

En élargissant le sens du rituel pour y inclure des coutumes qui n'avaient peu ou pas de rapport avec la magie et la religion et qui pouvaient être créées autant à la ville qu'à la campagne, les anthropologues et les ethnologues ouvraient le champ de l'étude des rituels dans les sociétés modernes ${ }^{11}$. Il devenait alors possible d'entreprendre l'observation des rituels du mariage au $\mathrm{xx}^{\mathrm{e}}$ siècle dans une perspective ville/campagne. Ce travail a été entrepris au Québec par Denise Lemieux et Lucie Mercier à partir de sources autobiographiques. Elle ont remarqué la transformation des rituels, provoquée par les changements économiques et sociaux liés à l'urbanisation. La diffusion de nouveaux rites modifie à la fois le scénario et le sens des rituels du mariage au cours de la première moitié $\mathrm{du} \mathrm{xx}^{\mathrm{e}}$ siècle ${ }^{12}$. Peter Ward a constaté l'amorce de ce processus dans les villes du Canada anglais au cours de la seconde moitié du XIx ${ }^{\mathrm{e}}$ siècle; favorisés par l'anonymat de la ville et par la progression de l'individualisme, les jeunes ont commencé, au cours de cette période, à se libérer de la mainmise de l'Église et de la famille ${ }^{13}$.

Les pratiques rituelles ne sont pas demeurées insensibles aux changements économiques et sociaux : nous voulions apprécier l'ampleur de l'introduction des innovations en comparant les rituels de la vallée du Haut-Richelieu avec ceux d'autres régions du Québec. La dynamique culturelle favorise-t-elle uniquement le transfert des coutumes de la ville vers la campagne? La population de la campagne qui se déplaçait vers la ville a amené avec elle les pratiques qu'elle connaissait et elle les a adaptées à son

10. Édouard-Zotique Massicotte, «De la durée des noces», Bulletin des recherches historiques, 36,7 (1930) : 392; "Une noce populaire d'il y a cinquante ans », L'Almanach du peuple (Montréal, Beauchemin, 1925), 334-340; sœur Marie-Ursule, Civilisation traditionnelle des Lavalois (Québec, Presses universitaires Laval, 1951), 118-130.

11. Martine Segalen, Rites et rituels contemporains (Paris, Nathan, 1998), 20-26.

12. Denise Lemieux et Lucie Mercier, Les femmes au tournant du siècle. Âges de la vie, maternité et quotidien (Québec, Institut québécois de recherche sur la culture, 1989), 135-136.

13. Peter Ward, Courtship, Love, and Marriage in Nineteenth-Century Canada (Montréal, McGillQueen’s University Press, 1990), 171-172. 
nouvel environnement. Malgré cela, certains rituels ont pu disparaitre à la suite de l'étiolement des réseaux de parenté, trop étendus dans l'espace. La ritualité ne sert toutefois pas seulement à exprimer la force des relations de parenté et de voisinage. Les rituels ont pu émerger parmi les pairs (groupes d'âge, collègues de travail, etc.), comme ils peuvent avoir été accomplis dans l'intimité de la famille immédiate ou du couple. Des échanges dynamiques entre la ville et la campagne, malgré une nette prédominance de la première, ont sans doute permis d'assurer une certaine continuité dans les pratiques rituelles au cours de ces décennies d'intense urbanisation. Cette relation ville/ campagne, fertile pour la compréhension des phénomènes économiques, a selon nous une valeur explicative intéressante en ce qui concerne les pratiques culturelles.

La question des différences entre la ville et la campagne mérite d'être étudiée pour une autre raison. Martine Segalen écrit, dans les dernières pages de son livre Amours et mariages de l'Ancienne France, qu' «il est aujourd'hui bien artificiel d'opposer les rites de la campagne à ceux de la ville, tant leurs relations sont étroites ${ }^{14}$ ». L'uniformisation des rituels du mariage qui résulte de l'avancée de la culture de masse, entendue ici au sens de pratiques culturelles diffusées par les médias, est indéniable, tant en France qu'au Québec ou ailleurs en Occident. Malgré tout, il nous semble important de vérifier s'il subsiste, à la fin du $\mathrm{xx}^{\mathrm{e}}$ siècle, un mode de vie différent dans la campagne du Haut-Richelieu, une région où l'influence de la métropole montréalaise s'est fait sentir dès le $\mathrm{xIX}^{\mathrm{e}}$ siècle. Des distinctions, significatives pour les témoins, devraient départager les rituels de la ville et de la campagne.

\section{LE TERRITOIRE ET LA MÉTHODOLOGIE DE L'ENQUÊTE}

\section{a. La vallée du Haut-Richelieu}

Notre enquête porte sur une région délimitée par les circonscriptions de Napierville, Saint-Jean et Iberville. Cette région présente l'avantage d'offrir un portrait contrasté pour l'observation des relations ville/ campagne. Dès le début du siècle, le noyau urbain Saint-Jean-Iberville se distingue de l'ensemble du Québec par le développement de son secteur industriel. En 1941, plus de $55 \%$ de la population active est employée dans les manufactures, alors que cette proportion dépasse légèrement $25 \%$ dans l'ensemble du Québec ${ }^{15}$. Dans les années 1940, les deux grands secteurs

14. Martine Segalen, Amours et mariages de l'Ancienne France (Paris, Berger-Levrault, 1981), 165.

15. La population active de 14 ans et plus classée par industrie est donnée pour la ville de Saint-Jean et pour l'ensemble du Québec dans le recensement de 1941. 
de l'activité industrielle sont la métallurgie et le textile. Toutefois, ce type d'entreprises ne domine pas complètement la structure industrielle de la ville : la transformation des produits agricoles, par exemple, est aussi un secteur important d'activités.

Par ailleurs, le territoire agricole qui entoure le centre urbain se distingue aussi du reste du Québec. Avant même que le marché montréalais absorbe leur production, les producteurs situés aux abords de la rivière Richelieu exportaient du foin et des chevaux vers les États-Unis. Quand le Québec amorce la spécialisation de sa production agricole, les agriculteurs de ces trois circonscriptions ont une longueur d'avance. Ils se sont orientés plus rapidement vers la commercialisation au début du siècle et plusieurs d'entre eux ont choisi des spécialités différentes des autres producteurs québécois. En 1981, ils se situent encore à l'avant-garde, tant par le choix de leur spécialité que par leurs revenus : ils dirigent de très grandes entreprises en comparaison avec les agriculteurs de l'ensemble du Québec.

\section{b. La méthodologie de l'enquête}

Nous avons réalisé 65 entrevues à Saint-Jean-Iberville et dans les villages de la région, auprès de témoins sélectionnés en fonction de différentes variables : l'origine rurale ou urbaine des époux, leur profession et celle de leurs parents, l'année du mariage. Les témoins ${ }^{16}, 35$ personnes ou couples mariés entre 1920 et 1940 et 30 mariés entre 1980 et 1995, ont été classés dans trois grandes catégories socioprofessionnelles : les agriculteurs, les travailleurs manuels et les travailleurs non manuels. Ces personnes devaient également s'être mariées dans une paroisse urbaine ou rurale, soit avant 1940, soit depuis 1980. Étant donné le cadre de cette recherche (études doctorales) et l'approche choisie (entrevues semi-dirigées exhaustives), ce nombre d'entrevues est assez élevé. Nous avons dû limiter le nombre de variables et restreindre à deux les périodes étudiées. Nous voulions que les deux périodes (avant 1940 et après 1980) soient éloignées le plus possible dans le temps, de manière à présenter deux moments très différents de la ritualité du mariage au $\mathrm{xx}^{\mathrm{e}}$ siècle. Le début de la Seconde Guerre mondiale et les changements sociaux et économiques radicaux qui ont suivi nous semblaient être une charnière incontournable pour l'étude des pratiques rituelles. Par ailleurs, le début des années 1980 coïncidait avec une nouvelle

16. Les témoins sont parfois des couples, plus souvent des personnes seules. 
ritualité, après les remises en question des années 1960 et 1970, comme l'ont constaté Denise Lemieux et Lucie Mercier ${ }^{17}$.

Au terme de l'enquête, nous devions avoir atteint le seuil minimal de dix entrevues pour chacune des segmentations (catégories socioprofessionnelles : agriculteurs, travailleurs manuels ou travailleurs non manuels; lieu de résidence : rural ou urbain $)^{18}$. Dans les faits, nous avons 23 mariages célébrés dans une paroisse rurale et 12 dans le centre urbain au cours de la première période; pour la seconde période, nous avons 10 mariages à la campagne et 20 à la ville. Les ethnologues et les anthropologues ont établi un seuil de saturation de l'information, atteint entre la $20^{\mathrm{e}}$ et la $30^{\mathrm{e}}$ entrevue $^{19}$. L'ajout de nouvelles entrevues rapporte très peu de données inconnues et s'avère peu rentable. Le nombre total d'entrevues que nous avons réalisées est donc suffisamment élevé pour permettre de stratifier les deux corpus et de comparer les sous-groupes entre eux.

L’informateur est considéré comme le témoin privilégié d’un événement dont l'histoire a gardé peu ou pas de traces documentaires. Son point de vue sur le sens du rituel est sollicité au même titre que tous les autres éléments de contexte qui entourent le déroulement des rites. Toutefois, sa conscience des gestes faits n'est jamais totale. La vision du chercheur englobe et dépasse la compréhension issue du sens commun. Les résultats de l'enquête sont mis en perspective avec d'autres observations de rituels semblables, retracés dans des contextes historiques différents, voire dans des cultures différentes. Le sens du rituel résulte de ces comparaisons et de ces mises en contexte ${ }^{20}$. Le sens des gestes rituels n'est pas élaboré par les individus dans leur for intérieur; il est construit socialement. Les rituels appartiennent à des univers culturels que l'histoire peut reconstituer.

17. Denise Lemieux et Lucie Mercier, «La formation du couple et ses rituels : l'analyse des changements de la période 1950-1980, à travers les récits de vie», dans Gilles Pronovost, dir., Comprendre la famille. Actes du 1er symposium québécois de recherche sur la famille (Québec, Presses de l’Université du Québec, 1992), 53-69.

18. Pour plus de précisions concernant la méthodologie, voir, Martine Tremblay, Les rituels du mariage dans la vallée du Haut-Richelieu au $\mathrm{Xx}^{e}$ siècle, indicateurs de la différenciation sociale et marqueurs culturels, thèse de doctorat (études québécoises), Université du Québec à Trois-Rivières, 1998, 326 p., chapitre 2, "Méthodologie de la recherche».

19. Jean-Pierre Deslauriers, Recherche qualitative. Guide pratique (Montréal, McGraw-Hill, 1991), 84; Rodolphe Ghiglione et Benjamin Matalon, Les enquêtes sociologiques. Théories et pratiques (Paris, Armand Colin, 1978), 50; Daniel Bertaux, "L'approche biographique. Sa validité méthodologique, ses potentialités ", Cahiers internationaux de sociologie, 69 (1980) : 209-210.

20. Pierre Bourdieu, op. cit., 49; et Clifford Geertz, The Interpretation of Cultures (New York, Basic Books, 1973), 20, ont souligné l'importance de la comparaison et de la mise en contexte dans l'analyse des données qualitatives. 
Notre méthode d'enquête par entrevue au moyen d'un questionnaire très élaboré ${ }^{21}$ vise à repérer systématiquement tous les éléments du rituel. Ainsi, les données recueillies sont non seulement pertinentes, mais elles sont comparables. Le rôle de l'enquêteur n'est pas négligeable dans le processus d'activation de la mémoire et de triage des données. Le premier stade de l'objectivation se passe là, sur le terrain, en présence de l'informateur. L'enquêteur doit faire la première critique de source à mesure qu'il reçoit l'information de son interlocuteur, de manière à pouvoir obtenir sa réaction sur-le-champ. Ce dernier peut ainsi corriger une erreur de chronologie ou régler tout autre problème soulevé par l'enquêteur.

Lorsque toutes les données relatives à une question sont placées l'une à la suite de l'autre et triées en fonction des variables à l'étude, les témoignages divergents ressortent du corpus. Il reste alors à expliquer ces divergences ou à écarter le témoignage incongru. Les données, agrégées en sous-groupes qui se recoupent partiellement, s'éclairent et se nuancent mutuellement (selon ce qu'on a appelé le principe de la triangulation interne $)^{22}$. La comparabilité des réponses constitue un élément fondamental dans le travail d'objectivation.

Enfin, la validité des choix méthodologiques que nous avons faits tout au long du travail de terrain est assurée par notre participation au groupe de recherche sur les Dynamiques culturelles interrégionales de l'IREP. Les décisions concernant la conduite de l'enquête y ont été discutées à plusieurs reprises. Nous avons également bénéficié des réflexions méthodologiques faites par d'autres chercheurs et étudiants menant des enquêtes de terrain sur les rituels du mariage (triangulation externe) ${ }^{23}$. Les réunions de cette équipe multidisciplinaire, de même que quelques rencontres avec des chercheurs français, ont été l'occasion de présenter les résultats de notre travail. C'est dans ce contexte d'interactions constantes et soutenues entre chercheurs et étudiants que nous avons élaboré nos stratégies de recherche.

L'approche morphologique retenue, c'est-à-dire l'observation de la configuration externe du rituel ${ }^{24}$, a permis d'éclairer certaines caractéristiques du lien entre l'individu et la société. Chaque expérience vécue par les témoins est unique; elle converge néanmoins vers des coutumes qui distinguent des groupes sociaux et des milieux de vie. Ces expériences sont

21. Nous présentons l'outil d'enquête à l'annexe 1 de notre thèse de doctorat.

22. Alex Mucchielli, Dictionnaire des méthodes qualitatives en sciences humaines et sociales (Paris, Armand Colin, 1996), 60.

23. Ibid.

24. Gérard Bouchard, «L'analyse morphologique de la culture...», op. cit., 641. 
aussi situées dans le temps et dans l'espace; elles s'inscrivent dans le cours de l'histoire du Québec au $x^{\mathrm{e}}$ siècle et révèlent les idées qui sous-tendent la socialisation et le développement de l'identité des individus par le biais des relations familiales.

\section{LES DIFFÉRENCES VILLE/CAMPAGNE PENDANT LA PÉRIODE 1920-1940}

Les informations recueillies montrent qu'au cours des années 1920-1940, les parents de la campagne étaient préoccupés par les fréquentations de leurs enfants. Ils n'acceptaient que très rarement de laisser sortir les jeunes filles dans des lieux publics comme les salles de danse ou de cinéma. Ces dernières recevaient leur ami au salon, en présence des parents et des frères et sœurs. C'était d'ailleurs les rencontres familiales et la sociabilité de voisinage qui leur permettaient de rencontrer pour la première fois celui qui allait devenir leur époux.

Les femmes de la ville profitaient d'une plus grande liberté. S'il arrivait souvent qu'elles reçoivent leur ami au salon, elles pouvaient aussi le fréquenter dans les endroits publics. Mais les parents demeuraient vigilants. Les familles de la ville exerçaient une forte autorité sur leurs enfants, même si ces derniers étaient un peu plus libres dans leurs déplacements que les jeunes ruraux ${ }^{25}$. Malgré cette apparente liberté, les moments d'intimité entre les hommes et les femmes sont rares puisque les groupes d'amis relayent les parents. Les rites des fréquentations contribuaient à encadrer les rapports d'intimité et à fixer les rôles ${ }^{26}$.

Les rites qui se situent entre la demande en mariage et la cérémonie elle-même étaient peu importants chez les ruraux. L'annonce du mariage enclenchait les préparatifs de la noce, qui était célébrée dans les semaines suivantes. Les couples de la campagne étaient peu disposés à publiciser leur décision de se marier et ne consultaient pas leur groupe d'amis dans leur décision, non plus que leurs camarades de travail ${ }^{27}$. Les showers et

25. L'âge moyen au mariage est relativement plus élevé chez les témoins de la ville mariés entre 1920 et 1940 . Il est de 24,5 pour les hommes et de 21,5 ans pour les femmes de la campagne, alors que les hommes de la ville se marient, en moyenne, à l'âge de 26,3 ans et les femmes à 23,1 ans.

26. Les enquêtes menées par Denyse Baillargeon et Denise Girard à Montréal montrent aussi que les fréquentations étaient contrôlées grâce à une éducation morale très stricte. Denise Girard, Mariage et classes sociales. Les Montréalais francophones entre les deux Guerres (Sainte-Foy, Les Presses de l’Université Laval, 2000), 70-71; Denyse Baillargeon, Ménagères au temps de la crise (Montréal, Éditions du remue-ménage, 1991), 77.

27. D’autres enquêtes réalisées dans des milieux paysans démontrent aussi la rapidité des préparatifs et la quasi-absence de fiançailles, de shower et d'enterrement de vie de célibat. Marie-Josée 
enterrements de vie de garçon étaient rares, et les fiançailles, qui n'étaient pas fréquentes non plus, étaient intimes. Ces coutumes ne touchaient pas encore les familles rurales, qui ne ressentaient pas la nécessité de ritualiser la coupure entre le célibat et le mariage.

À la ville, au contraire, la décision du mariage donnait lieu à des rites qui incluaient la famille et les amis. Les fiançailles étaient l'occasion d'un repas qui réunissait les deux familles. Les rites du shower et de l'enterrement du célibat, organisés par la famille pour réunir les amis, étaient aussi plus fréquents et plus complexes qu'à la campagne ${ }^{28}$. Le mariage prochain des femmes était souligné à la maison et impliquait le don de cadeaux. Pour les hommes, la fête se déroulait plutôt dans les hôtels où l'on buvait à la santé du futur marié. Ces festivités soulignaient à la fois le changement de statut et les solidarités entre personnes de même sexe.

La journée de noces à la campagne était chargée de rites. Chez la plupart des agriculteurs, les parents de la jeune femme et ceux du jeune homme recevaient à tour de rôle la parenté des époux pendant la journée des noces. Ces repas étaient généralement suivis de chants, de musique et de danses. Tard dans la soirée, chacun retournait chez soi. Les mariés qui avaient décidé de faire un voyage de noces partaient le lendemain ou même quelques jours après la fête. La visite de la parenté, très souvent aux États-Unis, constituait le but de leur voyage. Là-bas, ils étaient reçus comme des rois : repas somptueux, danses et cadeaux. Au retour, les parents organisaient généralement un repas avec les frères et sœurs. Puis ces derniers, de même que les oncles et les tantes, invitaient les nouveaux mariés à venir les visiter. L'entrée des jeunes couples dans la vie conjugale était bien enserrée par la sociabilité familiale.

Huot, Les pratiques rituelles entourant le mariage dans les régions du Saguenay et de Charlevoix, mémoire de maîtrise (études régionales), Université du Québec à Chicoutimi, 1991, 55-60; Anne-Marie Desdouits, "Les rituels du mariage paysan sur la Côte-de-Beaupré et dans la Beauce», dans G. Bouchard avec la collaboration de S. Courville, La construction d'une culture. Le Québec et l'Amérique française (Sainte-Foy, Les Presses de l’Université Laval, 1993), 318-319.

28. Ces nouveaux rites apparaissent aussi bien dans une petite ville comme Saint-Jean que dans une ville moyenne comme Trois-Rivières ou une grande ville comme Montréal. Denise Girard, op. cit., 73-74; Marie-Josée Boisvert, Les rituels du mariage des ouvriers de Trois-Rivières, 1925-1940, mémoire de maîtrise (études québécoises), Université du Québec à Trois-Rivières, 1996, 61-63, 70-74. 
TABLEAU 1

Nombre et type de repas selon le lieu de résidence des mariés/es (1920-1940)

\begin{tabular}{l|c|c||c}
\hline Nombre de repas & Rural & Urbain & Total \\
\hline Un repas & & & \\
$\quad$ chaud & 3 & 2 & 5 \\
froid & 9 & 9 & 18 \\
Deux repas & 11 & 0 & 11 \\
Aucun repas & 0 & 1 & 1 \\
\hline Total des entrevues & 23 & 12 & 35 \\
\hline
\end{tabular}

Ces rites paysans commençaient toutefois à changer. Les plus riches parmi les agriculteurs ont adopté quelques-unes des pratiques répandues à la ville. Ainsi, le deuxième repas de la journée de noces a été aboli ${ }^{29}$. Le seul repas pouvait être un simple buffet froid, servi avec un verre de vin, avant le départ des mariés en voyage de noces. Il n’y avait alors pas de chant, ni musique ni danse. Au retour, ces riches agriculteurs reprenaient cependant le cycle des réceptions en l'honneur des mariés.

À la ville, l'unique repas de noces était servi par les parents de la mariée, dans leur maison ou à l'hôtel. Ce repas, habituellement un buffet froid, donnait rarement l'occasion aux jeunes mariés et aux invités de danser. La fête était écourtée, de sorte que le rapprochement du couple avec la parenté était réduit au strict minimum. Plus important encore, cette noce ne portait plus, comme à la campagne, le sens d'une alliance entre les deux familles. Pour les urbains, faire la noce était une responsabilité financière entièrement dévolue aux parents des filles. Immédiatement après le repas, les époux partaient en voyage, pressés de commencer leur vie conjugale en toute intimité. Ils ne cherchaient pas non plus à voir la famille pendant leur voyage de noces. Quelques familles organisaient encore des petites fêtes au retour, mais les nouveaux couples ne visitaient guère les frères et

29. Les noces paysannes du Saguenay, de la Mauricie et des Bois-Francs commencent aussi à subir l'influence du mode de vie urbain dans les années 1920 et 1930, bien que le voyage de noces n’y soit pas aussi répandu que dans la vallée du Haut-Richelieu. On a observé, dans ces trois premières régions, que l'un des repas était assez souvent un petit-déjeuner ou un goûter. L'alternance des repas (ou goûters) chez les parents de l'un et l'autre conjoint était cependant la règle. Voir René Hardy, Gérard Bouchard et Anne-Marie Desdouits, «Les rituels du mariage dans les campagnes de la Mauricie/Bois-Francs et du Saguenay au $\mathrm{xx}^{\mathrm{e}}$ siècle : regards sur les spécificités régionales ", dans Yves Roby et Nive Voisine, dir., Érudition, humanisme et savoir. Actes du colloque en l'honneur de Jean Hamelin (Sainte-Foy, Les Presses de l’Université Laval, 1996), 267-292. 
TA B L E A U 2

\section{But du voyage de noces selon le lieu de résidence \\ des mariés/es (1920-1940)}

\begin{tabular}{l|c|c||c}
\hline But du voyage de noces & Rural & Urbain & Total \\
\hline Visiter la parenté & 11 & 3 & 14 \\
Faire du tourisme & 4 & 9 & 13 \\
Pas de voyage & 8 & 0 & 8 \\
\hline Total des entrevues & 23 & 12 & 35 \\
\hline
\end{tabular}

les sœurs, encore moins les oncles et les tantes. Bref, les époux de la ville étaient plus détachés de la sociabilité familiale que ceux de la campagne.

\section{LES DIFFÉRENCES VILLE/CAMPAGNE PENDANT LA PÉRIODE | 980 - | 995}

Le scénario de la formation du couple dans les deux dernières décennies du siècle est assez homogène. Les jeunes se rencontrent et se fréquentent très librement. La décision du mariage les entraîne dans un cycle de festivités et de préparation : shower, enterrements de la vie de célibataire et fiançailles sont des rites très répandus. Habituellement, les mariés organisent eux-mêmes la noce en invitant la famille et les amis pour un repas et une soirée. La musique, la danse et les jeux viennent clore cette journée de célébration. Le soir ou le lendemain du mariage, ils partent en voyage de noces. À leur retour, la vie quotidienne reprend son cours et les rites de la sociabilité familiale sont complètement disparus. Malgré cette homogénéité dans les principaux rites, il subsiste un écart entre la ville et la campagne. L'attitude des couples de la campagne est différente à propos du choix du conjoint, de la place de la parenté le jour des noces et des sommes qu'ils sont prêts à dépenser pour leur mariage.

Les jeunes agriculteurs ${ }^{30}$, contrairement aux couples de la ville, choisissent de préférence leur conjoint dans leur entourage et ils se fréquentent plus longtemps avant de se fiancer ${ }^{31}$. Ils se fiancent après plus de deux années de fréquentations, alors que le mariage est décidé et même planifié.

30. Les témoins de la campagne dans la période 1980-1995 sont presque exclusivement des agriculteurs ( 9 sur 10$)$.

31. La différence entre l'âge moyen au mariage est peu significative : à la campagne, les hommes se marient en moyenne à 24,1 ans et les femmes à 22 ans; à la ville, les hommes se marient à 23,9 ans et les femmes à 22,9 ans. 
TABLEAU 3

Laps de temps entre la première rencontre et le début des fréquentations selon le lieu de résidence des mariés/es (1980-1995)

\begin{tabular}{l|c|c||c}
\hline Laps de temps & Rural & Urbain & Total \\
\hline Moins de 2 semaines & 1 & 5 & 6 \\
Entre 2 semaines et 1 an & 3 & 8 & 11 \\
Plus d'un an & 6 & 7 & 13 \\
\hline Total des entrevues & 10 & 20 & 30 \\
\hline
\end{tabular}

Les couples de la ville, au contraire, se fiancent dès le début des fréquentations et laissent durer la période des fiançailles plus d'un an. Les ruraux sont aussi plus réservés face à ce rite d'engagement et résistent à son extension (repas, invitations, cadeaux) : ils préfèrent l'intimité à la célébration des fiançailles en grande pompe.

La gestion de leurs affaires est également visible à travers les rites : ils témoignent plus que les autres de l'importance d'un contrat notarié. La ferme est une entreprise et les modalités du partenariat entre les conjoints sont formalisées dans le contrat de mariage. Pour certains, le mariage est le moment de partager les avoirs, alors que d'autres veulent mettre les terres, les animaux et les équipements agricoles à l'abri d'un partage dans l'éventualité d'un divorce. Tous les couples d'agriculteurs qui possèdent une terre, ou qui envisagent d'en acheter une, discutent les termes de leur engagement matrimonial devant un notaire ${ }^{32}$.

TABLEAU 4

Durée des fréquentations au moment des fiançailles selon le lieu de résidence des mariés (1980-1995)

\begin{tabular}{l|c|c||c}
\hline Durée des fréquentations & Campagne & Ville & Total \\
\hline Deux ans et moins & 0 & 7 & 7 \\
Deux ans et demi et plus & 7 & 8 & 15 \\
Pas de fiançailles & 3 & 5 & 8 \\
\hline Total des entrevues & 10 & 20 & 30 \\
\hline
\end{tabular}

32. Un seul couple, dont l'époux est un ouvrier agricole, n'a pas signé de contrat parce que les conjoints n'envisageaient pas de s'établir comme agriculteurs. 
TABLEAU 5

\section{Partage du coût de la noce selon le lieu de résidence des mariés/es (1980-1995)}

\begin{tabular}{l|c|c||c}
\hline Partage des coûts & Campagne & Ville & Total \\
\hline Parents de la fille & 1 & 1 & 2 \\
Parents des deux époux & 5 & 8 & 13 \\
Parents des époux & 2 & 4 & 6 \\
et les mariés & 2 & 7 & 9 \\
Mariés seuls & 10 & 20 & 30 \\
\hline Total des entrevues & & & \\
\hline
\end{tabular}

Les jeunes couples de la campagne sont aussi davantage entourés de la parenté au moment du mariage. Les oncles et les tantes, les cousins et les cousines se rendent à la maison des mariés avant la cérémonie. Les mariés organisent la journée du mariage en pensant à la parenté : ils peuvent, par exemple, décider de s'épouser dans la matinée, afin de favoriser la présence des oncles et des tantes qui ont des animaux à traire. Les couples de la campagne préfèrent, plus souvent que ceux de la ville, planifier la séance de photographies avant la cérémonie, afin d'éviter de faire attendre les invités au moment du repas.

Les agriculteurs mariés entre 1980 et 1995 semblent plus disposés à faire des dépenses extraordinaires le jour de leur mariage, et cela est favorisé par la plus grande participation des parents aux frais de la noce. Ils engagent des musiciens professionnels ou semi-professionnels, alors que les couples de la ville louent les services d'un animateur avec sa discothèque mobile. Ils choisissent le gâteau aux fruits plus souvent que les couples de la ville, même s'il est beaucoup plus onéreux que le gâteau blanc. Ils

TABLEAU 6

Offre de boissons alcoolisées à volonté selon le lieu de résidence des mariés/es (1980-1995)

\begin{tabular}{l|c|c||c}
\hline Offre de boissons & Campagne & Ville & Total \\
\hline Oui & 8 & 5 & 13 \\
Non & 2 & 15 & 17 \\
\hline Total des entrevues & 10 & 20 & 30 \\
\hline
\end{tabular}


offrent plus souvent les boissons alcoolisées à volonté à leurs invités, tandis que les urbains paient seulement le cocktail au moment de l'accueil à la salle et le vin servi pendant le repas. Les choix faits par les couples d'agriculteurs démontrent qu'ils veulent faire de leurs noces une grande fête inoubliable.

\section{PERMANENCES, CHANGEMENTS ET VARIABILITÉ DES RITUELS DU MARIAGE AU XX' SIÈCLE}

\section{a. Les permanences : cérémonie religieuse, famille et parenté}

Nous avons limité notre étude aux rituels de la formation du couple qui comportent une célébration religieuse catholique, délaissant du même coup toutes les autres formes d'union qui ont cours à la fin du $\mathrm{xx}^{\mathrm{e}}$ siècle. Nous avons fait ce choix parce que nous voulions obtenir des données comparables pour les deux périodes étudiées. Nous avons donc décidé d'observer les rituels du mariage comportant une cérémonie religieuse, même si cette forme d'union régresse depuis la fin des années 1970. Le recul du mariage religieux est cependant un peu moins rapide que celui du taux de nuptialité, selon une étude réalisée par Jean-Paul Baillargeon à la fin des années $1980^{33}$.

La cérémonie religieuse est l'élément culminant du rituel et cette caractéristique ne change pas depuis les années 1920 jusqu'au milieu des années 1990. Même dans les rituels les plus complexes, où l'on observe un shower, un enterrement de la vie de célibat, des fiançailles, etc., la cérémonie religieuse représente le nœud du scénario. C’est devant l'autel, lorsque le prêtre reçoit leur consentement et prononce la formule "vous êtes maintenant mari et femme», que le couple se considère définitivement marié. La présence de la famille n'est pas nécessaire à l'union, la fête non plus. Dans les années récentes, la cérémonie religieuse a pris une telle ampleur qu'elle constitue le moment crucial de la journée des noces. Les témoins étaient d'ailleurs unanimes pour déclarer que la tension s'est relâchée après la sortie de l'église. Leurs justifications du choix du mariage

33. Jean-Paul Baillargeon, «Les mariages religieux, 1976-1985», Recherches sociographiques, 28,2-3 (1987) : 340-343. Il n’y a pas eu, à notre connaissance, d'études publiées depuis ce temps sur l'évolution démographique des mariages religieux. Toutefois, l'analyse des taux de nuptialité montre la décroissance rapide du nombre de mariages parmi le groupe des 20-24 ans, qui recouvre la moyenne de l'âge au mariage parmi nos informateurs de la période 1980-1995 (22,6 ans pour les femmes et 24 ans pour les hommes). Ces taux passent, chez les femmes, de $70 \%$ en 1980 à $23 \%$ en 1995 , et chez les hommes, de $61 \%$ en 1980 à $12 \%$ en 1995 . Voir Louis Duchesne, La situation démographique au Québec, bilan 1999, Rétrospective du $20^{\mathrm{e}}$ siècle (Québec, Bureau de la Statistique du Québec, Les Publications du Québec, 2000), 91. 
religieux apportent un éclairage intéressant sur les rapports qu'ils entretiennent avec l'Église. Même s'ils sont non pratiquants, la majorité d'entre eux ont utilisé un ou plusieurs des mots suivants pour expliquer cette décision : catholique, béni, sacrement, baptisé, religion ${ }^{34}$. La réponse d'un informateur reflète très bien les convictions de ce groupe : «Parce que je suis catholique. Je suis pas pratiquant, je vais pas à l'église toutes les semaines. Je suis croyant, je suis catholique. Moi j'avais dans mon idée de me marier, de fonder une famille, d'avoir des enfants ${ }^{35}$.»

La place des parents dans les rituels du mariage est demeurée centrale tout au long du siècle. Les rites sont orientés de manière à montrer la transformation du lien filial et la formation du lien conjugal. Dans les années 1920 et 1930, la très grande majorité des jeunes demandaient la permission de se marier à leurs parents et le jeune homme demandait officiellement la main de sa future épouse à son beau-père. La demande en mariage, comme d'autres rites tels l'entrée et la sortie de l'église, signifiait le passage de la jeune femme de la tutelle de son père à celle de son mari. Ce sont les pères qui amenaient leurs enfants à l'église et agissaient en tant que témoins au mariage. Seules la mort ou la maladie grave pouvaient les empêcher de tenir ce rôle. Les pères, souvent aussi les mères, occupaient la place d'honneur près des mariés au cours du repas. Les parents étaient les hôtes de la noce.

Au cours des années 1980 et 1990, les parents ne sont pas nécessairement les hôtes de la noce, surtout à la ville. Ils tiennent cependant un rôle de premier plan dans le scénario nuptial. Plusieurs jeunes hommes demandent la main de leur fiancée, et les couples qui cohabitent ${ }^{36}$ se séparent la veille du mariage pour retourner chez leurs parents respectifs. Ce rite doit être mis en relation avec la grande liberté qui caractérise les fréquentations des jeunes. Sauf exception, la reconfiguration du lien filial est déjà entamée depuis longtemps au moment du mariage. Le retour des cohabitants à la maison familiale rétablit in extremis l'autorité parentale et tente de compenser symboliquement pour le détachement presque déjà accompli avec la famille d'origine.

La place des parents est aussi très importante au moment de la cérémonie religieuse. Les époux se rendent à l'église avec leurs pères respectifs,

34. Soit 13 couples sur les 21 qui ont évoqué des croyances pour expliquer leur geste.

35. E53.

36. Le tiers des témoins rencontrés ont cohabité officiellement avant leur mariage, trois couples du milieu rural et huit du milieu urbain. Quelques autres n'ont pas cohabité, mais ils étaient très souvent ensemble, dans l'appartement de l'un des conjoints $(\mathrm{N}=5)$. 
souvent aussi avec leurs mères, et c'est avec ces derniers qu'ils y font leur entrée. Dans plusieurs mariages célébrés au cours des dernières décennies, cette entrée à l'église est fortement ritualisée et donne lieu à des embrassades entre parents et enfants au milieu de la nef, avant que le futur époux n'amène la future épouse jusqu'à l'avant de l'église. Cette scène illustre exactement ce qui est visé par les rituels du mariage : les parents acceptent de voir un de leurs enfants établir un lien conjugal plus puissant que le lien filial. Cette reconfiguration des liens familial et conjugal est opérée par l'accomplissement du rituel religieux.

La présence de la famille élargie n'est pas essentielle au déroulement de la cérémonie, mais rares sont les époux qui, au début du siècle, désiraient se marier dans la plus stricte intimité. La célébration d'un mariage en présence des seuls témoins et de quelques membres de la famille proche résultait le plus souvent de mésententes dans la famille, ou parce que les conjoints étaient connus et ne voulaient pas d'un attroupement devant l'église. L'Église a d'ailleurs toujours été réticente à favoriser ce genre de célébration; elle a plutôt encouragé le déploiement et le faste des cérémonies. Lorsque la famille annonçait qu'elle s'apprêtait à célébrer un "grand» mariage, la parenté s'empressait d'accepter l'invitation pour ne rien manquer de ces festivités familiales extraordinaires.

Le faste caractérise encore les cérémonies des années 1980 et 1990. Les couples mariés récemment peuvent ajouter beaucoup d'éléments à la cérémonie et en faire un véritable spectacle, avec des musiciens et des chanteurs, un décor conçu et réalisé par des professionnels, des textes choisis et lus par les mariés dans le but d'émouvoir les invités. Les déplacements des époux et leur disposition face à la nef au moment de l'échange des consentements, alors qu' ils demeuraient à la balustrade, dos à la nef, dans les décennies précédentes, soulignent bien que les invités ont aussi un rôle à jouer dans cette cérémonie. Ils sont bien sûr les témoins du mariage en représentant la communauté chrétienne; ils sont aussi à la fois les spectateurs et les acteurs de cette mise en scène.

La présence d'un public observant le déroulement de la cérémonie religieuse est implicitement recherchée et ce public est composé d'abord et surtout par les membres de la famille élargie. C'est pour eux que l'on organise la fête. Le mariage est une affaire de famille, autant dans les années 1920 et 1930 que dans les années 1980 et 1990. Par contre, les couples de la ville mariés au début du siècle évitaient parfois de convier la parenté, afin de pouvoir partir plus tôt en voyage. La noce était pour eux un écueil qui retardait le moment de leur intimité. La misère économique qui sévissait 
dans les années 1930 n'explique pas ce départ précipité des mariés; les facteurs économiques retardent le mariage ou font en sorte que l'on dépense moins au moment de l'achat des costumes et des victuailles, par exemple, mais ils ont très peu d'influence sur les éléments essentiels du scénario nuptial, comme l'a aussi constaté Denise Girard à Montréal ${ }^{37}$. Ainsi, la mode d'une noce écourtée se répand dans les riches familles d'agriculteurs, après avoir conquis aussi bien les familles des journaliers que celles des commerçants et des professionnels de la ville. Les témoins invoquent très rarement leur pauvreté pour justifier un départ rapide après la cérémonie, alors que les horaires des chemins de fer étaient au centre de leurs préoccupations.

La noce, à demi abandonnée pendant les années 1920 et 1930, se retrouve au cœur des rituels dans les années récentes. Si au début du siècle les époux de la ville s'évadaient de la noce pour se retrouver seuls, les couples mariés récemment ne sont plus pressés et veulent profiter de cette fête au maximum. Le mariage n'est pas pour eux, sauf exception, un rite d'initiation ou de passage vers l'âge adulte. L'évolution des rapports hommes/femmes vers une plus grande intimité avant le mariage a permis aux mariés de la période 1980-1995 de renouer avec la fête telle que la plupart des familles d'agriculteurs la concevaient. Ils invitent la famille élargie et dépensent sans compter. Ils accomplissent des rites qui signifient leur volonté de rompre avec la vie quotidienne par des débordements de la sensibilité et des manifestations sonores et visuelles extraordinaires.

\section{b. Les changements : commercialisation et cohabitation}

Quelques changements ont modifié la forme et le sens des rituels du mariage. La commercialisation autour du mariage a transformé l'aspect morphologique et, sans doute, le sens des rituels. À la fin du siècle, les rituels paraissent se complexifier sous l'impact de la commercialisation en même temps qu'ils s'uniformisent. Ils rompent de manière plus tranchée avec la vie quotidienne. Nous ne pouvons cependant déterminer la portée exacte de cette commercialisation sur le sens des rituels à cette étape de la recherche.

S'il est un changement qui a affecté profondément les rituels du mariage au cours du $\mathrm{xx}^{\mathrm{e}}$ siècle, c'est bien l'acceptation de la cohabitation comme forme possible d'union. C'est le choix de pouvoir éluder le rituel qui a le plus transformé l'organisation et le sens du rituel. Auparavant rite 
de passage obligatoire, le rituel du mariage est devenu un événement auquel on choisit de participer. Au cours de la première période, les fréquentations et l'intégration des nouveaux époux à la sociabilité familiale étaient relativement plus ritualisées. Aujourd'hui, ces deux séquences rituelles sont simples, sinon disparues ${ }^{38}$. Par contre, les couples mariés récemment témoignent de festivités avec les amis et la famille dans les semaines qui précèdent et surtout pendant la journée des noces.

Au début du siècle, lorsque le rituel du mariage était aussi rite de passage, les couples qui ne voulaient pas d'une grande noce se soumettaient à un rituel minimal. Ils se mariaient et partaient en voyage tout de suite après la cérémonie. Ils n'avaient cependant pas le choix d'accepter l'encadrement familial pendant les fréquentations. Surtout, ils ne pouvaient cohabiter; la cohabitation était une position intenable dans le contexte culturel de la période 1920-1940. Ceux qui ont témoigné, dans les années 1920 et 1930, d'un mariage sans aucune festivité sont peut-être ceux-là qui, aujourd'hui, choisiraient de ne pas se marier. À l'opposé, les couples qui, à la fin du $\mathrm{xx}^{\mathrm{e}}$ siècle, désirent se marier font une noce fortement ritualisée. Toutefois, leurs fréquentations et leur entrée dans la vie conjugale sont peu ou pas contrôlées par la famille. On y trouve donc très peu de rites.

\section{c. Variabilité des rituels au cours du siècle}

Nous sommes partie de l'idée qu'une forte autorité exercée par la famille et l'Église favoriserait le déploiement de rituels fastueux et relativement semblables d'un mariage à l'autre. D'où l'hypothèse que les rituels sont complexes et homogènes lorsque les institutions, les groupes d'âge et la famille ont beaucoup d'emprise sur les individus. Or, c'est tout le contraire que nous avons observé au cours de notre enquête. Au début du siècle, alors que les jeunes étaient bien encadrés par la famille et l'Église, la variabilité des rituels était très grande. Les familles avaient le choix de faire beaucoup ou très peu de festivités à l'occasion du mariage d'un de leurs membres.

Aujourd'hui, parmi ceux qui décident de ritualiser leur union, plusieurs rites sont presque incontournables. Si les couples paraissent pouvoir composer leur rituel selon leurs goûts, ils adhèrent presque tous à des rituels semblables et complexes. La très grande majorité des jeunes qui se marient témoignent des rites de la robe blanche, de l'ordre d'arrivée à

38. Les séquences rituelles sont des ensembles de rites qui forment un tout dans l'espace et dans le temps. Ces séquences rituelles ont également une logique propre. 
l'église, de la photographie des mariés, du cortège après la cérémonie, de la fête avec le lancer du bouquet et de la jarretière, du voyage de noces, etc. $\mathrm{Au}$ cours du siècle, les rituels du mariage sont devenus non essentiels et non obligatoires à la formation de l'union. Mais ceux qui persistent à y recourir ${ }^{39}$ rendent compte de rituels denses, complexes et diffusés uniformément dans toutes les couches de la population.

Ces jeunes qui choisissent aujourd'hui de ritualiser leur union affirment leur volonté de s'identifier aux personnes mariées de leur entourage, plus particulièrement à leurs parents. Ils cherchent dans cette identification un apaisement face à l'angoisse engendrée par le changement très rapide qui caractérise la société actuelle $\mathrm{e}^{40}$. En optant pour le mariage religieux, les époux des dernières décennies démontrent que le rituel agit sur leur propre insertion dans le déroulement du temps. Le rituel donne un sens à leur engagement conjugal et familial, qu'ils voudraient bien inscrire dans la durée. Plusieurs de nos témoins, de même que ceux et celles rencontrés par Denise Lemieux et Lucie Mercier ${ }^{41}$, Martine Segalen ${ }^{42}$ ou Laurence Hérault ${ }^{43}$, évaluent d'ailleurs leurs gestes en fonction de la tradition. La plupart d'entre eux tentent de se définir en conformité avec ce qu'ils considèrent comme un mariage traditionnel, alors que seulement quelquesuns veulent plutôt s'en démarquer.

Ce besoin d'ancrage identitaire qui se manifeste à travers les rituels du mariage est largement exploité par l'industrie. En introduisant une dimension commerciale dans les rituels actuels, l'industrie contribue à leur uniformisation. La publication de magazines spécialisés sur le mariage, la tenue d'un salon des mariés, l'organisation de défilés de mode dans lesquels les vêtements de noces tiennent une bonne part, etc., diffusent une image stéréotypée des rites. La publicité faite autour des mariages de stars ou de princes est une autre voie qui conduit à la massification des rites. On a pu constater un mouvement menant à l'homogénéité des rites au cours du $\mathrm{xx}^{\mathrm{e}}$ siècle. Il est cependant difficile de savoir dans quelle mesure il y avait variabilité des rites au cours des siècles passés.

39. Voir la note 33.

40. Georges Balandier, Sens et puissance (Paris, Presses universitaires de France, 1971), 109.

41. Denise Lemieux et Lucie Mercier, "La formation du couple et ses rituels... », op. cit., 53-69.

42. Martine Segalen, Du parquet au paquet. Évolution des rites de mariage dans le Poitou d'aujourd'hui (Centre d'ethnologie française, 1993), 18 p. (Texte inédit).

43. Laurence Hérault, "Transmettre ou construire un rite? Ou comment rendre compte de l'évolution des pratiques rituelles", dans Gérard Bouchard et Martine Segalen, dir., Une langue, deux cultures. Rites et symboles en France et au Québec (Sainte-Foy, Les Presses de l'Université Laval, 1997), 149-176. 


\section{URBANISATION, TRADITION ET MODERNITÉ}

La perspective ville/ campagne appliquée à la ritualité du mariage permet d'aborder les rapports entre la tradition et la modernité, même si, au départ, notre recherche ne tenait pas compte de cette question. Les témoins de la campagne mariés entre 1920 et 1940 paraissent ouverts à l'innovation. Ils sont fiers de témoigner de rites caractéristiques de la modernité comme l'usage de l'automobile, la photographie de noces ou le voyage de noces. Ces coutumes représentent à leurs yeux un progrès, même si les futurs mariés et leurs familles ne disposent que d'une seule automobile pour se rendre à l'église, même si la photographie de noces n'est pas prise par un photographe professionnel et même si le voyage de noces se déroule dans la parenté. À la lumière des entrevues réalisées avec des commerçants habitant les villages et des plus riches agriculteurs, nous pouvons avancer que ce sont eux qui adoptent les changements les premiers et qui contribuent à leur diffusion dans les campagnes ${ }^{44}$. La popularité de ces rites, qui sont des signes de la modernité, démontre la grande influence des innovations provenant de la ville.

D’autres rites caractéristiques de la modernité ont bouleversé les rituels paysans. La prédominance du mode de vie urbain est perceptible dans le raccourcissement du temps de la noce, dans le choix du samedi comme seule journée convenant à un mariage, dans la préférence accordée à la saison estivale, dans la mode du shower, etc. La diffusion de ces rites provenant de la ville a contribué à l'uniformisation des rituels au cours du siècle, bien qu'il reste aujourd'hui des différences entre la ville et la campagne. Mais l'ordre de transmission est parfois inversé. Les transferts culturels se font aussi de la campagne vers la ville, des familles d'agriculteurs vers les familles ouvrières. Les rites des fréquentations, par exemple, sont semblables partout, les parents de la ville ayant conservé une grande autorité sur leurs enfants. Il y a tout de même une nette prédominance de la ville dans cette dynamique culturelle et, dans l'état actuel de nos connaissances, le processus irréversible de l'urbanisation nous porte à croire que les différences qui subsistent entre la ville et la campagne sont des traces vouées à s'effacer lentement.

Pourtant, nous avons constaté que certains rites paysans, disparus à la ville au début du siècle, ont été réactivés dans les rituels des années 1980

44. Nous ne visions pas à vérifier cette hypothèse au début de la recherche. Notre échantillon comprend quatre familles de commerçants résidant dans l'un ou l'autre des villages et sept familles d’agriculteurs modérément fortunées ou aisées. 
et 1990. La fête, par exemple, avec son repas somptueux et ses nombreux invités, les décorations, la musique, la danse et les jeux, étaient observés à la campagne entre 1920 et 1940. Plusieurs caractéristiques de cette fête, comme le repas chaud et fastueux, la musique, la danse et les nombreuses invitations, sont disparues à la ville à la même époque, mais elles réapparaissent dans les rituels récents. D’autres pratiques, comme la grande demande exécutée par certains couples mariés récemment, ont aussi de quoi surprendre tant elles semblent inadaptées au contexte social actuel. Ces jeunes, qui aujourd'hui se fréquentent librement, acceptent de voir le jeune homme demander la main de la jeune femme au père de cette dernière. Dans ce cas précis, il semble que le sens du rite soit devenu accessoire, alors que la forme a été conservée et qu'elle s'est cristallisée.

La réinvention de rites aux origines très anciennes, comme les fiançailles, la bénédiction paternelle et le lancer de la jarretière, illustre bien une forme de transfert culturel de la campagne vers la ville, avec une rupture dans le temps. Les fiançailles existaient avant même l'ère chrétienne et elles ont été observées dans les traditions paysannes, de la France par exemple. Elles ont été interdites au début du xviII ${ }^{\mathrm{e}}$ siècle, ici au Québec comme partout ailleurs dans la chrétienté, là où les traditions locales permettaient de les abolir ${ }^{45}$. Au Québec, elles réapparaissent vers la fin du $\mathrm{XIX}^{\mathrm{e}}$ siècle $^{46}$, non pas dans la paysannerie mais dans les familles de la bourgeoisie, et elles sont assez répandues dans le noyau urbain de Saint-JeanIberville au cours de la période 1920-1940. Elles ont connu une large diffusion pendant le $\mathrm{xx}^{\mathrm{e}}$ siècle, si bien que la grande majorité des couples mariés récemment en témoignent ${ }^{47}$. Par ailleurs, la bénédiction paternelle, donnée aux futurs époux par leur père respectif avant de quitter la maison, est un rite paysan observé au Québec pendant le xix ${ }^{\mathrm{e}}$ siècle ${ }^{48}$. Ce rite, qui n’a pratiquement pas été relevé pour la période 1920-1940, apparaît comme une pratique bien implantée parmi les jeunes mariés récemment, autant à la ville qu'à la campagne ${ }^{49}$. Enfin, le lancer de la jarretière

45. Paul-André Leclerc, "Le mariage sous le régime français», Revue d'histoire de l'Amérique française, 13, (décembre 1959) : 385 ; André Burguière, «Le rituel du mariage en France : pratiques ecclésiastiques et pratiques populaires (xVI et xviII siècles", Annales ESC, 33,3 (1978) : 645.

46. Denise Lemieux et Lucie Mercier, Les femmes au tournant du siècle..., op. cit., 150.

47. Seulement 3 couples sur 10 à la campagne et 5 sur 20 à la ville ne se sont pas fiancés.

48. Édouard-Zotique Massicotte, «Une noce populaire... », op. cit., 334-340

49. Seulement trois filles d'agriculteurs ont été bénies par leur père respectif entre 1920 et 1940. Au cours de la période 1980-1995, dans le milieu rural, 7 épouses sur 10 et 4 époux sur 10 la reçoivent. À la ville, 14 épouses sur 20 et 7 époux sur 20 reçoivent la bénédiction de leur père respectif (parfois les deux parents). 
pendant ou après le repas de noces était inconnu au Québec au début du siècle. Différentes variantes de ce rite ont été relevées par les folkloristes dans plusieurs régions paysannes de la France au $\mathrm{XIX}^{\mathrm{e}}$ et au début $\mathrm{du} \mathrm{xx}^{\mathrm{e}}$ siècle $^{50}$. Ce rite de la jarretière est observé dans presque toutes les noces récentes dans la vallée du Haut-Richelieu.

Cette discontinuité des formes rituelles dans le temps et dans l'espace est une découverte tout à fait inattendue qui nécessite de nouvelles recherches.

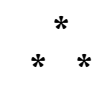

Pourquoi les jeunes couples mariés dans les années 1980 et 1990 se soumettent-ils à des rites révolus et très éloignés de leur vie quotidienne? Bien sûr, le rituel du mariage a surtout une fonction symbolique. Ces résurgences démontrent qu'il existe, parmi certains jeunes, un besoin de sacraliser des gestes et de les relier aux rituels accomplis par leurs parents. Mais encore? Comment expliquer ce processus par lequel des gestes répandus chez les paysans et tombés en désuétude dans les premières décennies du siècle peuvent ensuite être réactivés dans leur forme et dans leur sens par les urbains à la fin du siècle? Comment expliquer que ces transferts culturels n'aient subi ni la contrainte du temps ni celle de l'espace?

En attirant notre attention sur l'invention des traditions, Eric Hobsbawm propose une explication intéressante ${ }^{51}$. Apparue au début de la modernité dans le champ politique afin d’ancrer la légitimité des Étatsnations dans un passé immémorial, l'invention des traditions est un procédé qui a envahi toute la vie sociale. De plus, les rituels sont des gestes qui, comme les attitudes individuelles et les formes du langage, sont modifiés très lentement ${ }^{52}$. Cette permanence permet d'assurer la continuité des formes, sinon du sens, alors que d'autres activités produisent des changements très rapides.

En observant les pratiques sociales, on peut donc s'attendre à trouver des gestes hérités du passé, à plus forte raison dans le domaine de la ritualité, comme on peut aussi voir ressurgir des formes anciennes, réinventées pour répondre à des besoins nouveaux. Les gestes du mariage, anciens ou réin-

50. Arnold Van Gennep, Manuel de folklore français contemporain, 1,II : Du berceau à la tombe (fin) (Paris, A. et J. Picard, 1946), 519-521.

51. Eric Hobsbawm, «Introduction : Inventing Traditions», dans Eric Hobsbawm et Terrence Ranger, dir., The Invention of Tradition (Cambridge, Cambridge University Press, 1983), 1-14.

52. Georges Balandier, op. cit., 108-109. 
ventés, trahissent l'importance de la tradition dans une culture qui, en apparence, semble l'avoir rejetée. Si la cérémonie du mariage ne constitue plus un rite de passage obligatoire, ceux qui continuent d'y adhérer sont sans conteste attachés à ce qu' ils considèrent comme des traditions. Ces individus puisent à même le stock de rites connus. La diffusion massive des informations leur permet également d'avoir recours à des rituels anciens, ou même étrangers à leur culture, sans jamais les avoir observés dans la réalité. Ces "gestionnaires du passé», comme les nomme Balandier, peuvent ainsi raviver des rites oubliés, les redéfinir et les remodeler au besoin. Ils s'appuient à la fois sur la mémoire familiale et sur la culture de masse pour réactiver des pratiques anciennes de la culture instituée.

Dans le cas des rituels du mariage, ces individus trouvent un encadrement et un appui dans l'Église, qui donne une image stable et rassurante du mariage. En désirant la ritualisation de leur union, les couples accentuent délibérément leur perception de la continuité du mariage à travers le temps plutôt que la rupture provoquée par la cohabitation et les échecs matrimoniaux qu'ils voient autour d'eux. En ce sens, leur choix démontre qu'ils privilégient la continuité au changement. Si les urbains des années 1920 et 1930 considéraient les anciennes coutumes paysannes incompatibles avec leur nouveau mode de vie, ils sont nombreux dans les années 1980 et 1990 à renouer avec ces vieilles coutumes. Malgré le recul actuel des rituels du mariage dans la population en général, l'importance de ces "gestionnaires du passé » est encore assez grande pour tenter de les caractériser socialement et rechercher ce qu'ils peuvent avoir conservé des anciennes valeurs du monde rural. Les usages qu'ils font des coutumes illustrent la grande plasticité de la culture. 
CARTE 1

\section{Comtés et paroisses de la vallée du Haut-Richelieu et villes de Saint-Jean et d'lberville}

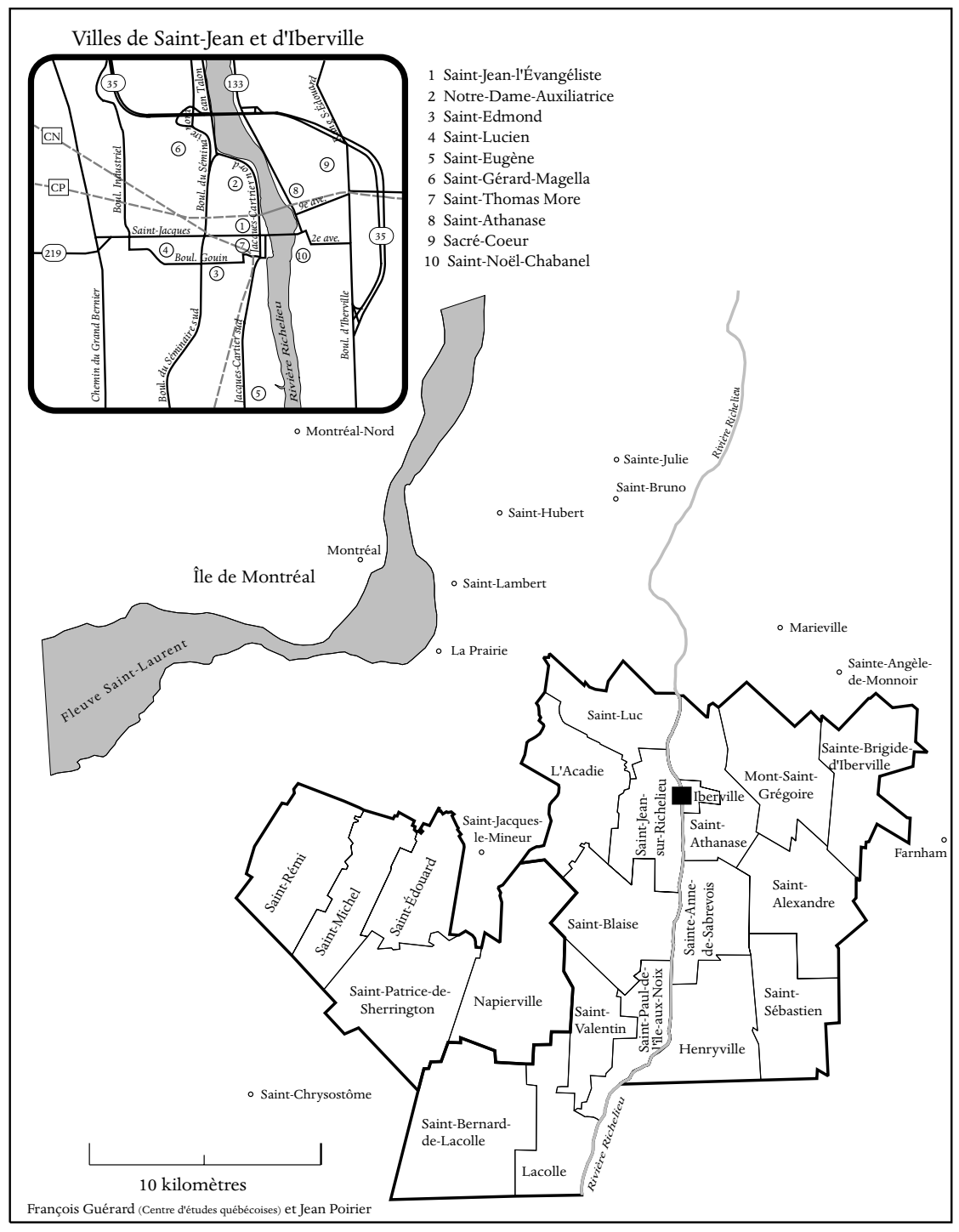

